\title{
Phonon-Assisted Excitation Transfer in Quantum Dot Molecules
}

\author{
E. RozBICKI AND P. MaChNiKOWSKi \\ Institute of Physics, Wrocław University of Technology \\ Wybrzeże Wyspiańskiego 27, 50-370 Wrocław, Poland
}

\begin{abstract}
We derive a quantum-kinetic description of phonon-assisted Förster transfer between two coupled quantum dots (a quantum dot molecule). We show that the exciton state decays to the ground state of the quantum dot molecule via a combination of the Rabi rotation and exponential decay. For moderately spaced dots this process takes place on a picosecond timescale.
\end{abstract}

PACS numbers: $63.20 . \mathrm{Kr}, 73.63 . \mathrm{Kv}$

\section{Introduction}

Coupled quantum dots (QDs), referred to as quantum dot molecules (QDMs), have attracted much attention in recent years [1]. Besides the electronic coupling or superradiance effects [2], the properties of QDMs are affected by phonon-related phenomena. In particular, many experiments have shown phonon-assisted excitation transfer [3] between the QDs. For moderate separations between the dots $(6 \mathrm{~nm})$ tunneling is exponentially suppressed and the energetically lowest states correspond to spatially direct excitons localized in individual QDs [4]. Such states are bound by the Coulomb interaction via interband dipole moments [5], i.e., by the Förster interaction [6]. Signatures of such coupling were indeed found in a photon-correlation experiment [7].

In this paper we derive a quantum-kinetic description of the evolution of an exciton in a QDM, including the effect of the carrier-phonon coupling. We show that, depending on the parameters, the system can show different dynamical scenarios, ranging from partial pure dephasing to an almost exponential transfer. As we shall see, the transfer may be very fast (on the timescales of several picoseconds), that is, $2-3$ orders of magnitude faster than suggested by the existing perturbative estimates [8]. 


\section{Theory}

We consider two flat, cylindrically symmetric, coaxial QDs, separated by the distance $D$ along the $z$ axis and interacting with phonons. The formalism will be restricted to the subspace, spanned by the states $|0\rangle,|1\rangle$, corresponding to a single exciton in the ground state of the lower and upper dot, respectively (with a fixed polarization). We assume that the wave functions of excitons confined in different dots do not overlap, so that no phonon-assisted transitions are possible. The Hamiltonian of the system is then

$$
H=\frac{1}{2} \Delta \sigma_{z}+V \sigma_{x}+\hbar \sum_{k} w_{k} b_{k}^{\dagger} b_{k}+\sum_{l=0,1}|l\rangle\langle l| \sum_{k}\left(g_{k}^{(l)} b_{k}+g_{k}^{(l) *} b_{k}^{\dagger}\right),
$$

where $\sigma_{i}$ are the Pauli matrices in the basis $(|0\rangle,|1\rangle), \Delta$ is the energy mismatch between the dots, $V$ is the amplitude of the Förster coupling, $b_{k}^{\dagger}, b_{k}$ are creation and annihilation operators for the phonon mode with a wave vector $k, w_{k}$ is the corresponding frequency, and $g_{k}^{(l)}$ are exciton-phonon coupling constants. For heavy-hole excitons confined in QDs stacked along $z$ one has $V=d^{2} /\left(4 \pi \epsilon_{0} \epsilon_{\mathrm{r}} D^{3}\right)=$ $(3 / 4)[\hbar c /(D E)]^{3} \hbar \Gamma$, where $d$ is the interband dipole moment, $\epsilon_{0}$ is the vacuum dielectric constant, $\epsilon_{\mathrm{r}}$ is the relative dielectric constant of the crystal, $c$ is the speed of light, and $\Gamma$ is the inverse exciton lifetime.

The most effective interaction between neutral excitons and phonons is the deformation potential coupling to longitudinal acoustic phonons. Approximating the exciton wave function by a product of identical Gaussians one gets [9]

$$
g_{k}^{(0,1)}=\left(\sigma_{\mathrm{e}}-\sigma_{\mathrm{h}}\right) \sqrt{\frac{\hbar k}{2 \rho v u}} \exp \left(-\left(l k_{\perp} / 2\right)^{2}-\left(l_{z} k_{z} / 2\right)^{2}\right) \exp \left( \pm \mathrm{i} k_{z} D / 2\right),
$$

where $\sigma_{\mathrm{e}}, \sigma_{\mathrm{h}}$ are the deformation potential constants for electrons and holes, $v$ is the normalization volume for phonons, $k_{\perp}, k_{z}$ are the components of the wave vector in the $\mathrm{QD}$ plane and along $z, l_{\perp}, l_{z}$ are the confinement sizes in these two directions, and $u$ is the speed of sound.

The evolution of the interacting carrier-phonon system is described using the correlation expansion technique [10-12]. One starts from the three dynamical variables $x, y, z$ describing the carrier state, $x=\left\langle\sigma_{x}(t)\right\rangle, \ldots$, where $\sigma_{i}(t)=\mathrm{e}^{\mathrm{i} H t / \hbar} \sigma_{i} \mathrm{e}^{\mathrm{i} H t / \hbar}$ are the operators in the Heisenberg picture. From the Heisenberg equations of motion one finds the dynamical equations for these three variables,

$$
\dot{x}=\mathrm{i}\left\langle\left[H, \sigma_{x}\right]\right\rangle=-\Delta y-4 y B_{k}-4 y y_{k},
$$

and analogously for $y$ and $z$ (from now on, the time dependence will not be written explicitly). Obviously, this set of equations is not closed, but involves the new phonon variables $B_{k}=g_{k}\left\langle b_{k}\right\rangle$, as well as phonon-assisted variables of the form $y_{k}=g_{k}\left\langle\left\langle\sigma_{y} b_{k}\right\rangle\right\rangle=\left\langle\sigma_{y} b_{k}\right\rangle-\left\langle\sigma_{y}\right\rangle\left\langle b_{k}\right\rangle$. Here $g_{k}=\left(g_{k}^{(0)}-g_{k}^{(1)}\right) / 2$ and the double angular brackets, $\langle\langle\ldots\rangle\rangle$, denote the correlated part of a product of operators, obtained by subtracting all possible factorizations of the product. 
Next, one writes down the equations of motion for the new variables that appeared in the previous step, for instance,

$$
\begin{aligned}
\dot{y}= & \mathrm{i}\left\langle\left[H, y_{k}\right]\right\rangle=\Delta x_{k}-2 V z_{k}-\mathrm{i} w_{k} y_{k}+\left|g_{k}\right|^{2}(\mathrm{i} y z+x) \\
& +2 \sum_{q}\left(x_{q k}+\tilde{x}_{q k}\right)+4 x_{k} B_{q}+2 x \sum_{q}\left(B_{q k}+\tilde{B}_{q k}\right),
\end{aligned}
$$

where the new two-phonon and two-phonon-assisted variables are defined as

$$
\begin{aligned}
& B_{q k}=g_{q} g_{k}\left\langle\left\langle b_{q} b_{k}\right\rangle\right\rangle, \quad \tilde{B}_{q k}=g_{q}^{*} g_{k}\left\langle\left\langle b_{q}^{\dagger} b_{k}\right\rangle\right\rangle, \\
& x_{q k}=g_{q} g_{k}\left\langle\left\langle\sigma_{x} b_{q} b_{k}\right\rangle\right\rangle, \quad \tilde{x}_{q k}=g_{q}^{*} g_{k}\left\langle\left\langle\sigma_{x} b_{q}^{\dagger} b_{k}\right\rangle\right\rangle, \text { etc. }
\end{aligned}
$$

In the next step, one writes the equation of motion for these new variables, introducing three-phonon variables. It is clear that the resulting hierarchy of equations is infinite and has to be truncated at a certain level. Here we do this by setting all the correlated parts of three-phonon and three-phonon-assisted variables equal to zero. This amounts to neglecting three-phonon processes (that is, emission or absorption of three or more phonons within the memory time of the phonon reservoir, which is of order of $1 \mathrm{ps}$ ). The motivation for this procedure is that higher order correlations should play a decreasing role in the dynamics. From the equations of motion it is also clear that such higher order correlations develop in higher orders with respect to the coupling constants $g_{k}$.

In this way we treat the problem at the same level as in the recent work [12], taking into account the coherent and non-equilibrium phonons which are essential for the correct description of carrier phonon-kinetics in QDs. As an improvement over the standard approach [11, 12], we define collective, frequency-dependent variables

$$
\begin{aligned}
& B_{1}(\omega)=\sum_{k} \delta\left(\omega-\omega_{k}\right) B_{k}, \quad B_{2}\left(\omega, \omega^{\prime}\right)=\sum_{q k} \delta\left(\omega-\omega_{k}\right) \delta\left(\omega^{\prime}-\omega_{k}\right) B_{q k}, \\
& x_{1}(\omega)=\sum_{k} \delta\left(\omega-\omega_{k}\right) x_{k}, \quad x_{2}\left(\omega, \omega^{\prime}\right)=\sum_{q k} \delta\left(\omega-\omega_{k}\right) \delta\left(\omega^{\prime}-\omega_{k}\right) x_{q k}, \text { etc. }
\end{aligned}
$$

All the equations of motion can be rewritten in terms of these frequency-dependent variables. For instance, Eqs. (2) and (3) read

$$
\begin{aligned}
\dot{x}= & -\Delta y-4 y B_{1}(\omega)-4 y_{1}(\omega), \quad \dot{y}_{1}(\omega)=\Delta x_{1}(\omega)-2 V z_{1}(\omega)-\mathrm{i} \omega y_{1}(\omega) \\
& +J(\omega)(\mathrm{i} y z+x)+4 x_{1}(\omega) \int \mathrm{d} \omega^{\prime} \operatorname{Re} B_{1}\left(\omega^{\prime}\right) \\
& +2 \int \mathrm{d} \omega^{\prime}\left[x_{2}\left(\omega^{\prime}, \omega\right)+\tilde{x}_{2}\left(\omega^{\prime}, \omega\right)\right]+2 x \int \mathrm{d} \omega^{\prime}\left[B_{2}\left(\omega^{\prime}, \omega\right)+\tilde{B}_{2}\left(\omega^{\prime}, \omega\right)\right]
\end{aligned}
$$

where $J(\omega)=\sum_{k}\left|g_{k}\right|^{2} \delta\left(\omega-\omega_{k}\right)$. In this way, the set of variables labeled by points in the 3-dimensional reciprocal space is replaced by a set labeled by points on a real frequency axis. For the calculations, the frequency axis is discretized and the ordinary differential equation for the resulting variables is integrated numerically 
(we use 601 uniformly spaced points up to the frequency cut-off at $20 \mathrm{ps}^{-1}$, which yields $5.8 \times 10^{6}$ variables).

\section{Results}

Let us begin the presentation of the results with the noninteracting case, $V=0$. The occupation of each dot is then conserved, so that no excitation transfer may take place. Nonetheless, this does not mean that no carrier-phonon kinetics takes place. In Fig. 1 we present the evolution after an instantaneous preparation of the state $|+\rangle=(|0\rangle+|1\rangle) / \sqrt{2}$ in a pair of QDs with $\Delta=2 \mathrm{meV}$. The pure dephasing effect, related to the lattice response to the appearance of a charge distribution [13], reduces the coherence of the superposition state. This is also manifested by the decreasing amplitude of oscillations of the projection on the initial state, $\langle+|\rho(t)|+\rangle$, where $\rho(t)$ is the reduced density matrix of the carrier subsystem.

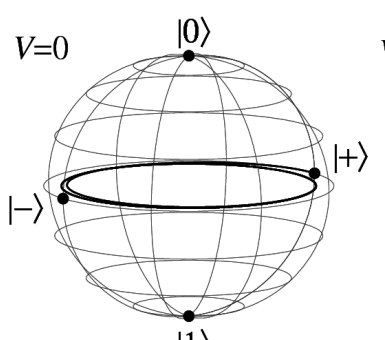

(a)

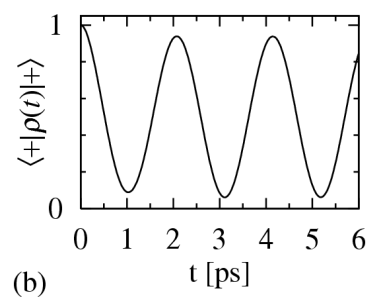

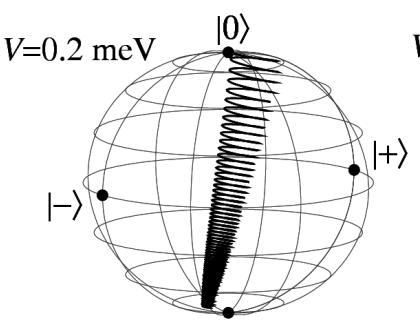

(c)

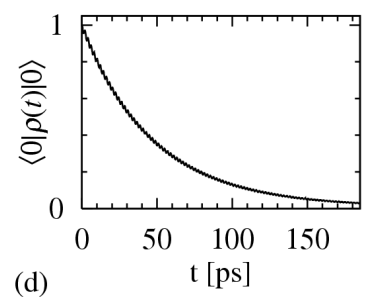

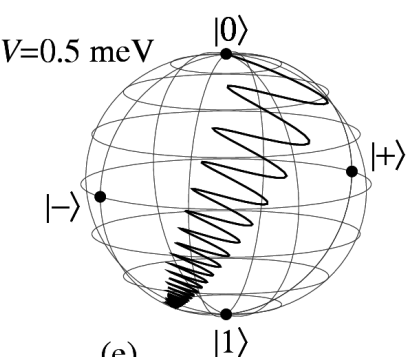

(e)

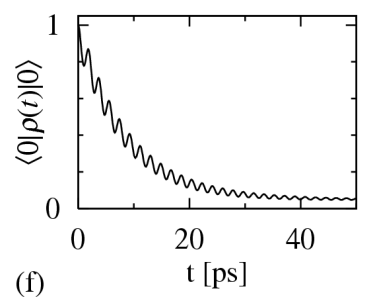

Fig. 1. (a,c,e) The Bloch sphere representation of the evolution in the three cases described in the text. (b) The overlap of the system state with the initial state $|+\rangle$. $(d, f)$ The occupation of the higher energy state as a function of time for two values of the Förster interaction. Here $l_{\perp}=4.5 \mathrm{~nm}, l_{z}=1 \mathrm{~nm}, D=6 \mathrm{~nm}$ and the temperature $T=0$.

In the presence of an interaction, $V \neq 0$, the occupations of the two dots are not conserved and excitation transfer becomes possible. As can be seen in Figs. 1c, e, the system evolution is a combination of a rotation around a tilted axis, defined by the eigenstates of the unperturbed Hamiltonian, and dephasing 
resulting from the interaction with phonons. As a result of the latter, at $T=0$ the system relaxes towards the lower eigenstate of the unperturbed Hamiltonian (with some correction due to phonon-induced energy shifts and polaron effects). For $V \ll \Delta$, this lower eigenstate is close to the state $|1\rangle$. In this limit, the transfer is nearly exponential, as can be seen in Fig. 1d.

When the interaction gets stronger the final state gains more admixture of the higher-energy dot. Now, the transfer takes place via clearly marked oscillations about an exponential trend. The value of $V=0.5 \mathrm{meV}$ used in Figs. 1e,f corresponds to the interband dipole moment of $9 e \AA$ (or a radiative lifetime of $470 \mathrm{ps}$ ) in the dipole approximation, which is within the range of typical values for self-assembled structures. For this value, the decay rate is about $10 \mathrm{ps,} \mathrm{which}$ means that the process is very fast.

The results presented in this paper show that the phonon-assisted excitation transfer in QDMs is an efficient process that may considerably affect the observable properties of these systems. For moderately spaced dots, the Förster coupling results in a fast transfer showing a pattern of oscillations around an exponential curve. We believe that these characteristic signatures should make it possible to identify this process in experimental observations.

\section{Acknowledgments}

P.M. is grateful to V.M. Axt for many discussions on the correlation expansion.

\section{References}

[1] M. Bayer, P. Hawrylak, K. Hinzer, S. Fafard, M. Korkusinski, Z.R. Wasilewski, O. Stern, A. Forchel, Science 291, 451 (2001); G. Ortner, M. Bayer, A. Larionov, V.B. Timofeev, A. Forchel, Y.B. Lyanda-Geller, T.L. Reinecke, P. Hawrylak, S. Fafard, Z. Wasilewski, Phys. Rev. Lett. 90, 086404 (2003); H.J. Krenner, M. Sabathil, E.C. Clark, A.F. Kress, D. Schuh, M. Bichler, G. Abstreiter, J.J. Finley, Phys. Rev. Lett. 94, 057402 (2005).

[2] M. Scheibner, T. Schmidt, L. Worschech, A. Forchel, G. Bacher, T. Passow, D. Hommel, Nature Phys. 3, 106 (2007); A. Sitek, P. Machnikowski, Phys. Rev. $B$ 75, 035328 (2007).

[3] R. Heitz, I. Mukhametzhanov, P. Chen, A. Madhukar, Phys. Rev. B 58, R10151 (1998); A. Tackeuchi, T. Kuroda, K. Mase, Y. Nakata, N. Yokoyama, Phys. Rev. $B$ 62, 1568 (2000); G. Ortner, R. Oulton, H. Kurtze, M. Schwab, D.R. Yakovlev, M. Bayer, S. Fafard, Z. Wasilewski, P. Hawrylak, Phys. Rev. B 72, 165353 (2005); T. Nakaoka, H.J. Krenner, E.C. Clark, M. Sabathil, M. Bichler, Y. Arakawa, G. Abstreiter, J.J. Finley, Phys. Rev. B 74, 121305(R) (2006).

[4] B. Szafran, S. Bednarek, J. Adamowski, Phys. Rev. B 64, 125301 (2001).

[5] B.W. Lovett, J.H. Reina, A. Nazir, G.A.D. Briggs, Phys. Rev. B 68, 205319 (2003); K.J. Ahn, J. Förstner, A. Knorr, Phys. Rev. B 71, 153309 (2005).

[6] T. Förster, Ann. Phys. (Leipzig) 2, 55 (1948); D.L. Dexter, J. Chem. Phys. 21, 836 (1953) 
[7] B.D. Gerardot, S. Strauf, M.J.A. de Dood, A.M. Bychkov, A. Badolato, K. Hennessy, E.L. Hu, D. Bouwmeester, P.M. Petro, Phys. Rev. Lett. 95, 137403 (2005).

[8] A.O. Govorov, Phys. Rev. B 71, 155323 (2005).

[9] K. Roszak, A. Grodecka, P. Machnikowski, T. Kuhn, Phys. Rev. B 71, 195333 (2005).

[10] F. Rossi, T. Kuhn, Rev. Mod. Phys. 74, 895 (2002).

[11] J. Förstner, C. Weber, J. Danckwerts, A. Knorr, Phys. Rev. Lett. 91, 127401 (2003); A. Krügel, V.M. Axt, T. Kuhn, P. Machnikowski, A. Vagov, Appl. Phys. $B$ 81, 897 (2005).

[12] A. Krügel, V.M. Axt, T. Kuhn, Phys. Rev. B 73, 035302 (2006).

[13] P. Machnikowski, V.M. Axt, T. Kuhn, Phys. Rev. B 75, 052330 (2007). 